\title{
Computational Base Substitution Analyses on Secondary Structure of Aptamer: Conformational Changes Diminish Complex Formation
}

\author{
Thangavel LAKSHMIPRIYA ${ }^{1,2, *}$, Subash Chandra Bose GOPINATH ${ }^{2,3}$, \\ Uda HASHIM ${ }^{2}$, Suresh Venkata CHINNI ${ }^{4}$ and Thean-Hock TANG ${ }^{1}$ \\ ${ }^{I}$ Advanced Medical \& Dental Institute, Universiti Sains Malaysia, Kepala Batas, Penang, Malaysia \\ ${ }^{2}$ Institute of Nano Electronic Engineering, Universiti Malaysia Perlis, 01000 Kangar, Perlis, Malaysia \\ ${ }^{3}$ School of Bioprocess Engineering, Universiti Malaysia Perlis, 02600 Arau, Perlis, Malaysia \\ ${ }^{4}$ Department of Biotechnology, Faculty of Applied Sciences AIMST University, 08100 Bedong, Malaysia
}

('Corresponding author's e-mail: lakshmipriya50@gmail.com)

Received: 10 April 2016, Revised: 23 February 2018, Accepted: 28 March 2019

\begin{abstract}
Factor IX (FIX) is an important protein in the blood clotting cascade, playing a key role. Previously, an anti-FIX RNA aptamer (34 mer) was generated to block blood coagulation, in order for it to be used as a substitute for currently available anti-coagulants. Bases in the loop region of this RNA aptamer have mainly involved the binding of FIX. Changes in 2 bases were found to diminish the complex formation with FIX, which could be predicted by evaluating the alteration in the secondary structure of the aptamer. In this study, computational analyses were carried out on the secondary structure analysis with aptamer, and the possible changes observed. It was confirmed that both A10 and A12 are the key bases involved in the complex formation with FIX. Similar structural analysis may helpful in identifying and predicting the importance of RNA bases in maintaining the secondary structure and their binding affinity.
\end{abstract}

Keywords: BayesFold, mfold, aptamer, factor IX, secondary structure

\section{Introduction}

Blood coagulation is the process in which blood changes from a liquid state to a gel form. The blood coagulation cascade involves 2 pathways, namely, intrinsic and extrinsic. The coagulation steps involved in these pathways have differences, but both involve working towards the production of Factor $\mathrm{X}$ and then catalyzing prothrombin to thrombin in the presence of calcium ion. Thrombin finally cleaves the circulating plasma protein fibrinogen to fibrin [1-7]. Regarding cardiovascular and peripheral vascular diseases, involvement of anti-clotting factors is a serious issue. Currently, heparin and warfarin have been used as anti-coagulant agents. However, heparin has been found to give side effects, and it is therefore necessary to generate a molecule that has an anti-coagulating effect while being less or nonimmunogenic.

Aptamers (artificial antibodies) are one of the suitable candidates for the aforementioned, expected to be ideal molecules due to their characteristics, such as stability, non-immunogenicity, and ease of preparation and modification. Aptamers are rare functional nucleic acid motifs elected by procedures involving isolation from a random library of nucleic acid molecules that have high affinity to the target by the iterative rounds of selection and amplification processes called 'SELEX', the systematic evolution of ligands by exponential enrichment. The basic scheme for this SELEX process is simplified and shown in Figure 1(a). By exploiting the SELEX method, previously, several aptamers have been selected for a wide variety of targets, including simple ions, small molecules, peptides, proteins, organelles, and even for entire cells. Interestingly, the isolated aptamers have displayed a high specificity and affinity to 
http://wjst.wu.ac.th

targets, which are comparable to the affinities achieved by antibodies against the appropriate antigens. Aptamers have been exploited in the past for various applications both in vitro and in vivo, including the detection and quantification of analytes, the inhibition of the function or activity of proteins (decoy strategy), the imaging process, and gene regulation [8-12]. Developing a specific aptamer as a probe would have a great impact on not only the development of specific diagnostic reagents, but also aid in the development of tailor-made drugs. Aptamers could be subjected to several kind of modifications to make them resistant to ribonucleases; also, the average sizes of the selected aptamers are small $(<6000$ daltons), compared to the antibodies.
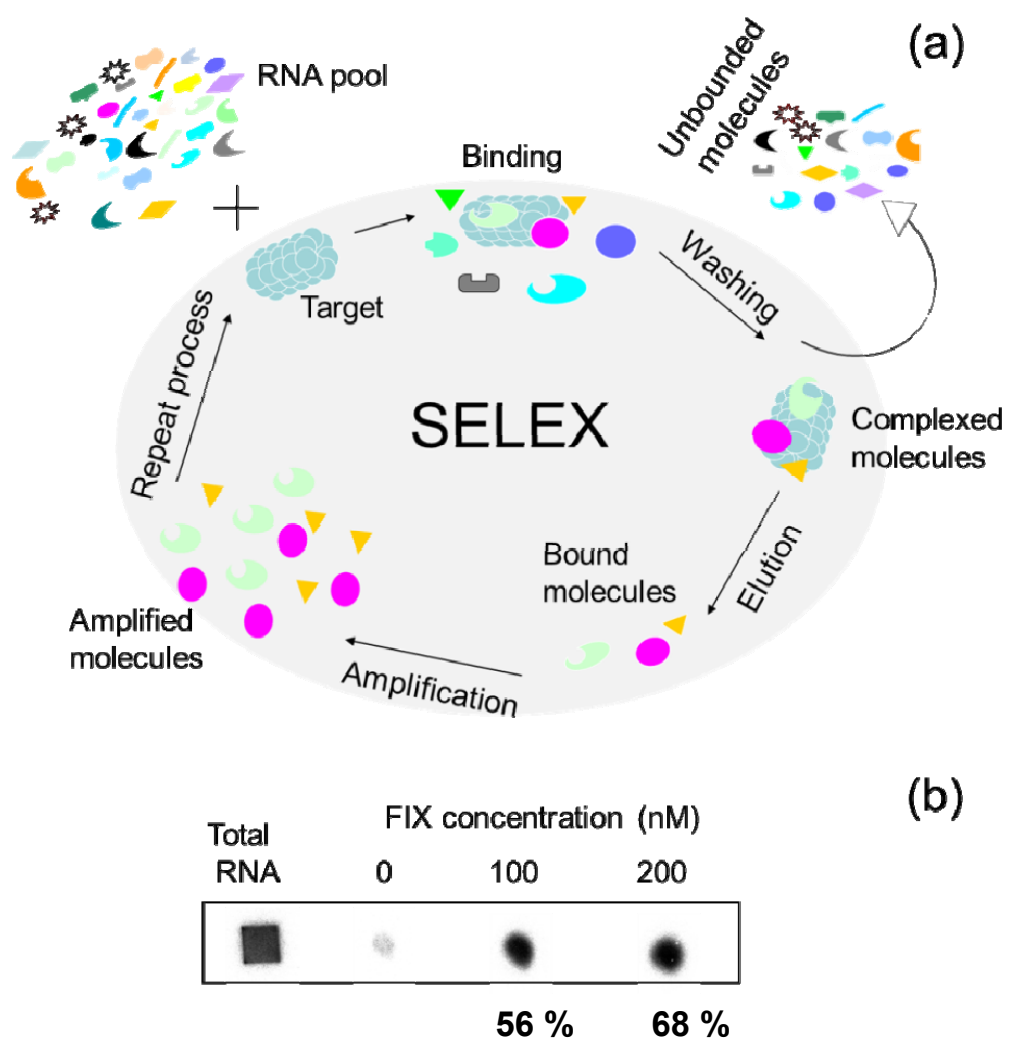

(b)

Figure 1 (a) Scheme for the SELEX process. A strategy for aptamer generation involves steps including complex formation, separation, and amplification; (b) Filter binding assay for aptamer and FIX binding. Different concentrations of FIX were used and tested against radio isotope-labelled aptamer. The label ' 0 ' indicates the binding of tRNA in the absence of the target.

Various works have proposed different aptamers for use against clotting proteins. Among these, anti-thrombin and anti-FIX aptamers are the important in their behavior as anti-coagulants [13]. Rusconi et al. [14] developed an RNA aptamer against FIX, which can act as an anti-coagulant and can be a substitute for heparin. This RNA aptamer is able to bind both FIX and FIXa, and has a higher stability under various conditions, as this RNA aptamer is modified by 2'fluro. The selected RNA aptamer has been found to have an important binding loop with the involvement of bases. It is expected that changes in the bases of loop regions will cause losses in binding. In this work, we demonstrate the possible alterations in the secondary structure of the aptamer upon changes in the bases of loops. The adjustments made by the structures to these changes were monitored by BayesFold or mfold software [15-20]. 


\section{Materials and method}

\section{Preparation of anti-Factor IX aptamer}

Human factor IX was purchased from American Diagnostica, Inc., USA. The 34-mer stable RNAaptamer, previously selected for use against factor IXa [14], was enzymatically synthesized by in vitro transcription using T7 RNA polymerase on a synthetic DNA template. The template, with the T7 promoter region (5'-AGTAATACGACTCACTATAGGGATGGGGACTATACCGCGTAATGCTG -3'), was synthesized to generate double-stranded DNA. Using this basic template DNA and the primers (forward 5'-AGTAATACGACTCACTATAGG-3'; reverse 5'- ATGGGGAGGCAGCATTACGCGG TATA-3'), we prepared the dsDNA by PCR, which now contained a T7 promoter (in italics). The PCR reaction mixture yielded the final conentrations $10 \mathrm{mM}$ Tris- $\mathrm{HCl}(\mathrm{pH} 8.8), 50 \mathrm{mM} \mathrm{KCl}, 1.5 \mathrm{mM} \mathrm{MgCl}$, $0.1 \%$ Triton X-100, $5 \mathrm{U}$ of Taq DNA polymerase (Takara), and $0.4 \mu \mathrm{M}$ of each primer was used. The reaction mixture was cycled at $94{ }^{\circ} \mathrm{C}$ for $1 \min 10 \mathrm{~s}, 55^{\circ} \mathrm{C}$ for $50 \mathrm{~s}$, and $72{ }^{\circ} \mathrm{C}$ for $1 \min 10 \mathrm{~s}$ for 10 cycles. The PCR product was precipitated and used for RNA preparation by in vitro T7 transcription. Transcription was performed at $37{ }^{\circ} \mathrm{C}$ overnight by using a DuraScribe transcription kit (Epicentre Biotechnologies, USA). Afterwards, the products were treated with $2 \mathrm{U}$ of DNase I (RNase free) for 10 min at $37^{\circ} \mathrm{C}$, to remove the template DNA, and were mixed with an equal volume of $2 \mathrm{X}$ urea buffer $(7 \mathrm{M}$ urea, $50 \mathrm{mM}$ EDTA, and $90 \mathrm{mM}$ Tris-borate containing $0.05 \%$ bromophenol blue). The reaction mixtures were denatured at $90{ }^{\circ} \mathrm{C}$ for $2 \mathrm{~min}$ and fractionated on a $15 \%$ polyacrylamide gel containing 7 $\mathrm{M}$ urea. The RNA band was excised, and the RNA was eluted from the gel as described before [20]. The RNAs were concentrated by vacuum, re-dissolved in water, and quantitated by absorbance at $260 \mathrm{~nm}$.

\section{Analysis of aptamers and binding assay}

To evaluate the aptamer binding with the target, the PCR product of aptamer was internally labeled using $0.5 \mathrm{mCi} / \mathrm{ml}\left[\alpha_{-}{ }^{32} \mathrm{P}\right] \mathrm{CTP}$ by in vitro transcription using T7 RNA polymerase on a synthetic DNA template. In vitro transcription was performed at $37^{\circ} \mathrm{C}$ for $3 \mathrm{hr}$ with a T7 Ampliscribe kit (Epicentre Technologies). Binding studies were performed using a filter binding assay. We used $20 \mathrm{nM}$ labeled RNA and 100 or $200 \mathrm{nM}$ of FIX. After the aptamer was incubated with the target protein at room temperature for $10 \mathrm{~min}$, the aptamer-FIX protein complexes were filtered through a pre-wetted nitrocellulose acetate filter (HAWP filter, $0.45 \mu \mathrm{m}, 13 \mathrm{~mm}$, Millipore) fitted in a "Pop-top" filter holder (Nucleopore) and washed with $1 \mathrm{ml}$ binding buffer $(10 \mathrm{mM}$ Hepes- $\mathrm{KOH}, \mathrm{pH} 7.4)$ and air dried, and the radioactivity quantitated with an image analyzer (Fuji Film). To ensure that the binding was specific, we performed binding assays in the presence of a 10-fold molar excess of tRNA as a nonspecific competitor. Experiments were performed in triplicate.

\section{Prediction of secondary structures}

The possible secondary structures of the aptamer were predicted using the mfold online soft (http://mfold.rna.albany.edu/?q=mfold) and also compared with BayesFold (http://www.ncrna.org/ KnowledgeBase/Bioinformatics/structure prediction/bayes fold/?searchterm=None). For the secondary structure prediction, parameters were defined for loop free energy decomposition, and other default parameters were followed.

\section{Results and discussion}

Prediction of secondary structures is an important step, as it implies the involvement of stems and loops in the binding events. With the secondary structure, stem(s) regions give stability, whereas loop(s) are involved in interactions with the target [21-23]. Here, we predicted the possible secondary structures for the RNA aptamer generated against FIX, an important factor in blood clotting. This anti-factor IX was originally generated by Rusconi et al. [14], and this group continued research on this aptamer; the aptamer is now undergoing clinical trial. Gopinath et al. [24] found that this aptamer utilizes extrinsic pathways and is able to bind FIX and FIXa. Due to this importance, herein, we performed structural prediction analyses as a model to predict the losses in the binding. Before structural analyses were 
http://wjst.wu.ac.th

conducted, we evaluated the binding ability of the prepared wild type aptamer using radio isotope labeling, and the aptamer-FIX complex was tested by the filter binding assay as reported elsewhere [25]. We used 2 different concentrations of FIX (100 and $200 \mathrm{nM})$ in the presence of tRNA as the competitor. We could visualize the genuine high affinity between aptamer and FIX in a dose-dependent manner. At $100 \mathrm{nM}, 56 \%$ labeled aptamer was bound, whereas $200 \mathrm{nM}$ showed $68 \%$ binding. No significant retention of the selected RNA occurred on the filter in the absence of FIX protein, indicating non-specific filter binders did not occur (Figure 1(b)). Previously, for anti-FIX aptamer, the dissociation constant was found to be $300 \mathrm{nM}$ by filter binding assay and $100 \mathrm{pM}$ and $37 \mathrm{pM}$ using waveguide mode sensor and surface plasmon-based biacore system, respectively. It was also found that this aptamer had good binding affinity; it was 25 times higher than in antibody binding with FIX protein [24].

Previously, Rusconi et al. [14] found that 2 bases (A10 and A12) in the loop region were necessary for the potential binding of this aptamer with FIX (Figure 2(a)). Using these binding bases as the key, we analyzed all the possible confirmation changes on the structure upon making mutations on A10 and A12. For these analyses, we considered 4 types of sequences for secondary structure prediction, viz., (i) wild type, (ii) A10 replaced by G10, (iii) A12 replaced by G12, and (iv) both A10 and A12 replaced by G10 and G12. With these alterations, the possible predicted structures were found by BayesFold or mfold software.

It was determined that the binding constants for the aptamer against factor IX and factor IXa were $418 \mathrm{pM}$ and $365 \mathrm{pM}$, respectively. These values infer that the complex formation between the aptamer and factor IX/IXa occur around $400 \mathrm{pM}$. Under this concentration, the binding of the aptamer and factor IX/IXa is saturated. However, when the sequence gets mutated or involves base substitution, there will be conformational changes that influence the complete loss in binding. Based on previous studies [14,24], AUA in the loop region is the main contact point with factor IX for complex formation. Upon mutation in these bases, there will be conformation changes in the aptamer secondary structure.

(a)

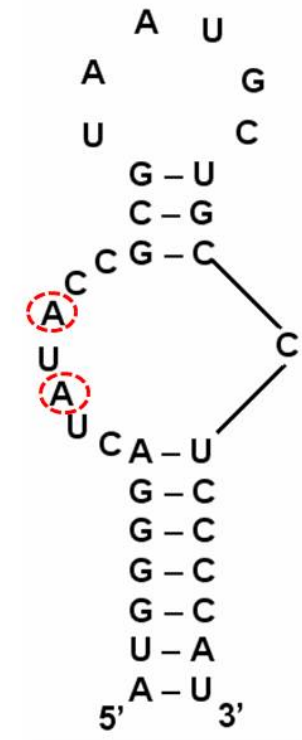

(b)

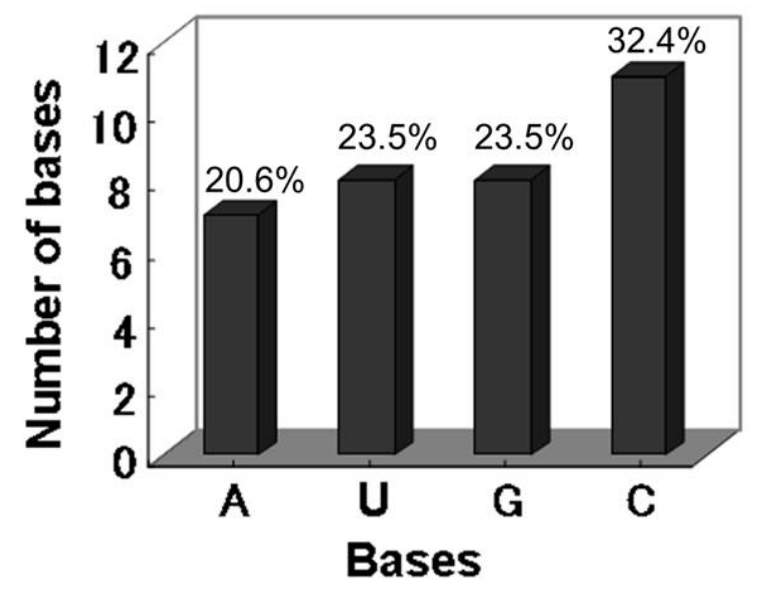

Figure 2 (a) Predicted secondary structure for anti-FIX aptamer, (b) Graphical representation of the probability of bases of anti-FIX aptamer. Calculation was made with the number of $\mathrm{A}, \mathrm{U}, \mathrm{G}$, and $\mathrm{C}$ bases. $\mathrm{C}$ base represents the highest frequency. 


\section{Analyses on wild type aptamer}

The wild type aptamer sequence (5' AUGGGGACUAUACCGCGUAAUGCUGCCUCCCCAU 3') includes all 4 bases viz., A, U, G, and C. Among these, C has a higher frequency, as it is repeated 11 times, followed by $\mathrm{U}$ and $\mathrm{G}$, which are both repeated 8 times. The fourth base $\mathrm{A}$ is repeated 7 times. These bases represent 32.4, 23.5, and $20.6 \%$, respectively (Figure 2(b)). In predicting the secondary structure of the wild type aptamer, we can predict 10 different structures having both stem and loop. It is clearly seen that, in all the structures, the bases AUA are in the loop region, and it is already proven that these are important regions in maintaining binding affinity with the FIX protein. As stated elsewhere, generally, RNA molecule may bind with the target in the loop region, so the loop region is important in maintaining the structure. All these structures (1 to 10) retain at least 2 loops with their structure. However, 4 structures, namely, 3, 5, 7, and 8, have 3 loops; further, all 10 structures have strong base stem regions (Figure 3).

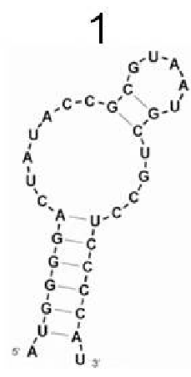

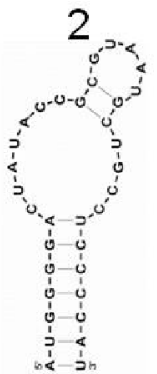

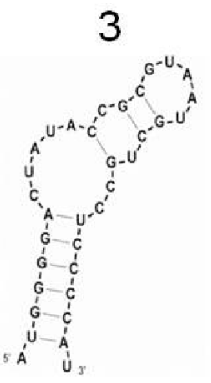

4

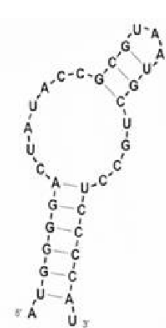

9

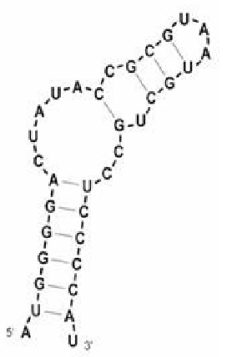

8

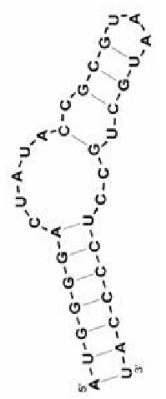

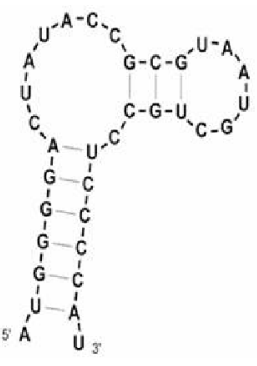

5

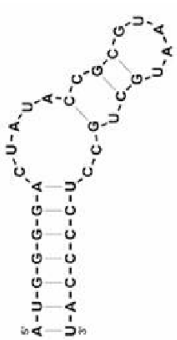

10

Figure 3 Possible structure predictions for wild type aptamer. Ten structures were predicted. Structures have either 2 or 3 loops and a strong base stem. The parameter defined for loop free energy decomposition and basic parameters were followed as described previously [26].

With this wild aptamer, it was already proven that, when the bases A10 and A12 in the loop region are changed to G10 and G12, they lose their binding affinities [14]. Generally, when antidote is added to the aptamer, the aptamer will bind with the antidote and lose its ability, but when the A10 \& A12 bases are changed independently or together, it may lose its binding ability with the antidote. The antidote stated in this study is a complementary sequence to the region of aptamer sequences. Upon complementation, the function or complex formation of the aptamer will be reverted. So, further structural analyses were carried out with the changes in these 2 bases, either by single mutation or double mutation. 


\section{Base substitution on A10 to G10}

In the loop region on RNA structure, which could bind to FIX, it is expected to have losses in binding when making the base substitution from A10 to G10. Figure 4 shows the possible predicted structure upon changing the base A10 to G10. With the obtained 10 possible structures, it was interesting to notice that, upon changing A10 to G10, the loop region become stem. Interestingly, in all 10 structures, A10 is in the stem region. Moreover, structures $1-8$ form 3 loops instead of 2 loop regions in the wild type. These 2 changes (forming a stem and 3 loops) seem to cause loss in the binding. However, structures 9 and 10 keep 2 loop structures, but G10 remains in the stem region.
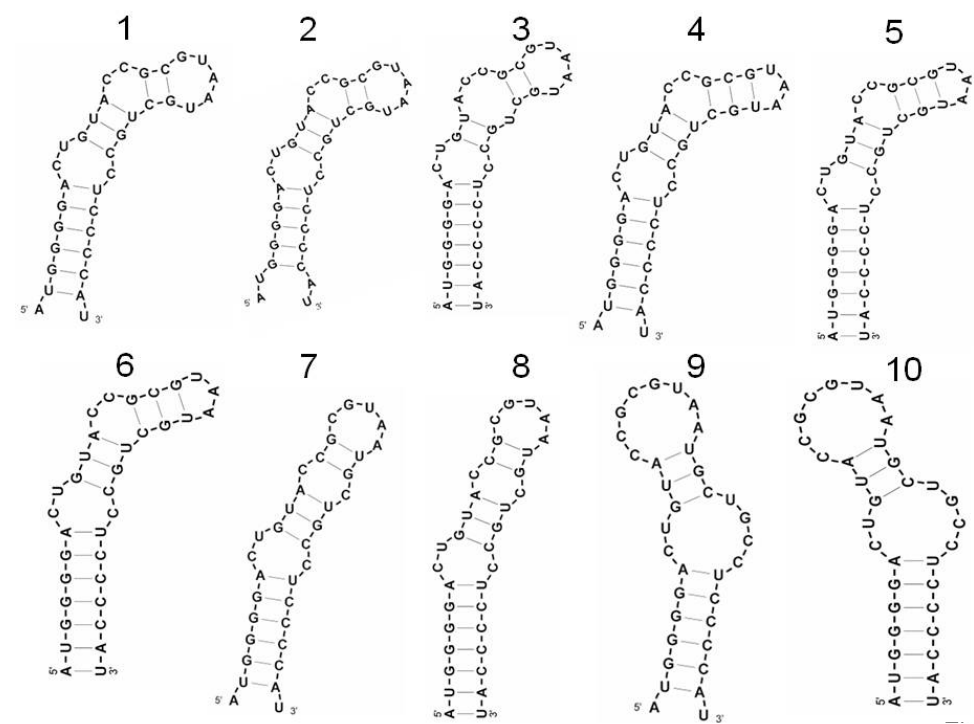

Figure 4 Possible structure predictions with A10 base substitution to G10. Ten structures were predicted. Structures have either 2 or 3 loops and strong base stem.

\section{Base substitution on A12 to G12}

Similar to the above analysis, we also made a base substitution on A12 and changed the A12 base into G12, as suggested in the mutation analysis by Rusconi et al. [14]. Upon this change, we could predict about 8 secondary structures, instead of the 10 structures predicted in the other cases. It was expected that, with these changes, there would be losses in the binding. As predicted in the A10 base substitution, in A12 base substitution, also all structures showed structural changes from the loop region to the stem in the G12 position. Most of the structures ( 1 to 6 ) make 3 loops with a strong base stem, whereas structures 7 and 8 form only 2 loops, keeping G12 at the stem region (Figure 5). Again, it is obvious that losses in the binding is due to formation of the stem with G10. The base substitution to make different secondary structures is caused by the loss in the main contact point with factor IX. Upon mutation in these bases, there will be conformation changes in the aptamer secondary structure and loss of the binding between the aptamer and factor IX. This loss in the binding is well discussed by Rusconi et al. [14]. In this study, the binding loss has been demonstrated by binding assay. 


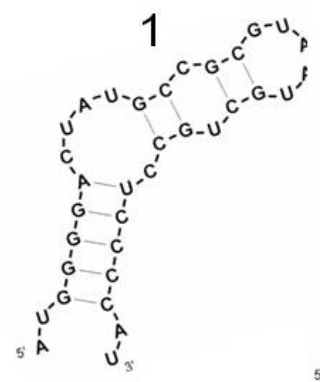

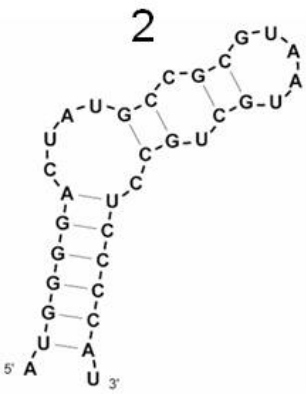
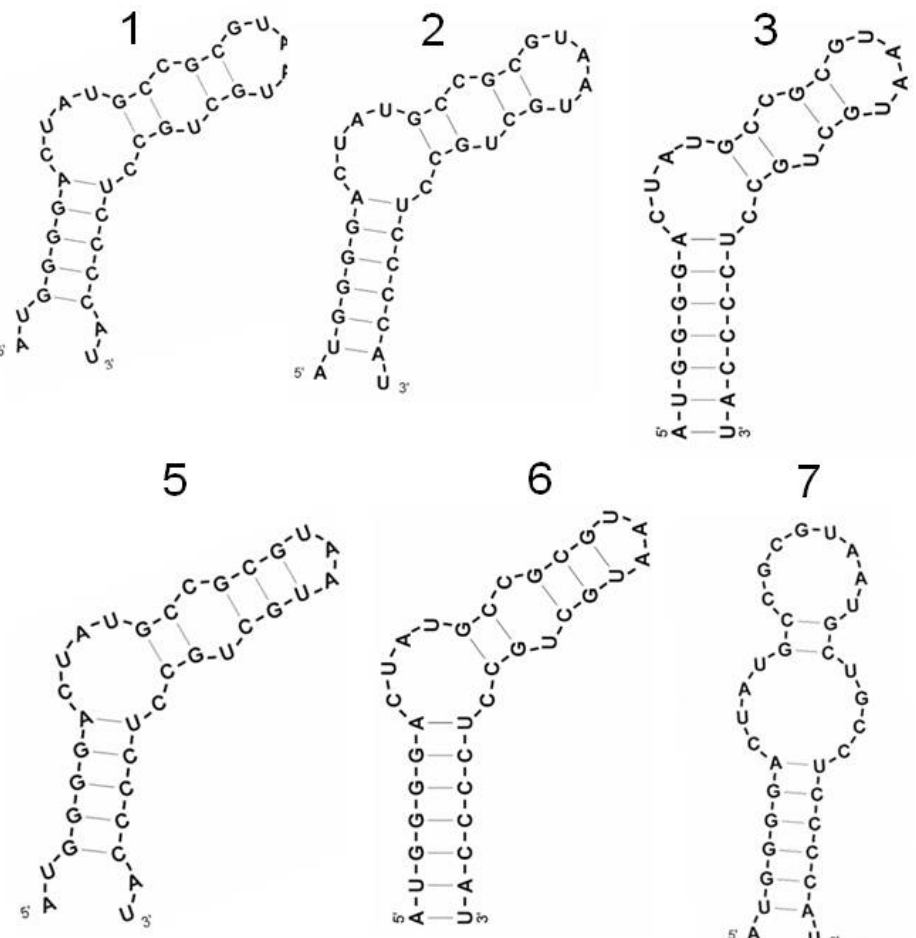

6

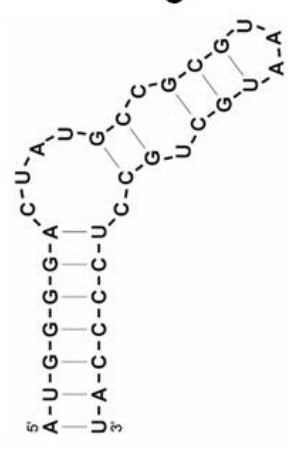

7

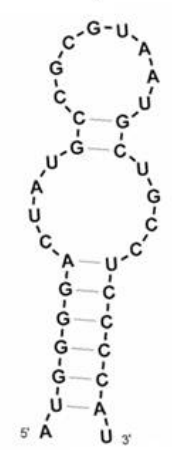

4

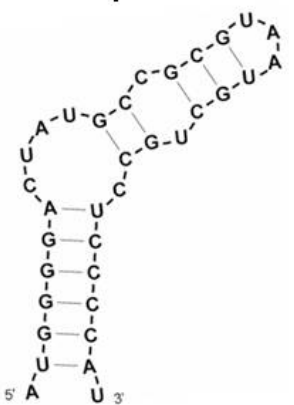

8

Figure 5 Possible structure predictions with A12 base substitution to G12. Eight structures were predicted. Structures have either 2 or 3 loops and strong base stem.

\section{Base substitution on A10 to G10 and A12 to G12}

From the above study, it is clear that both A10 and A12 are important to the anti-FIX aptamer to make the complex and to keep the secondary structure stable. To make sure about this prediction, we made further double mutations by substituting both A10 and A12 to G10 and G12. With the analyses made with a folding program, we could obtain about 10 structures. In most of the cases, the region of substitutions tended to form unstable regions of short stems and loops (Figure 6). However, it was obvious that the total structure was changed compared to the wild type, which probably caused loss in the binding. There was no structure with only 2 loops, as observed in other cases above. This double mutation analysis confirms the above analyses. 


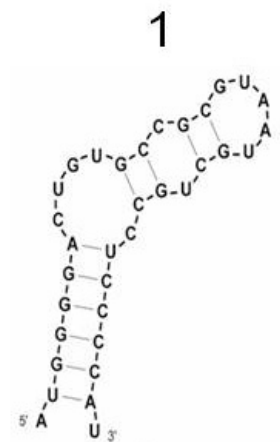

6

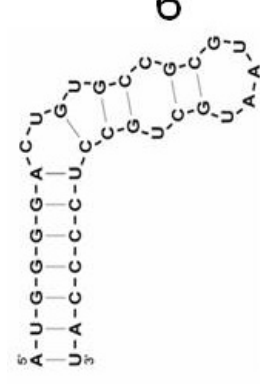

\section{2}

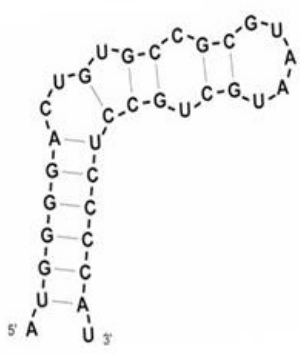

\section{7}

3

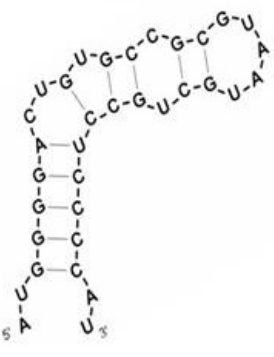

4

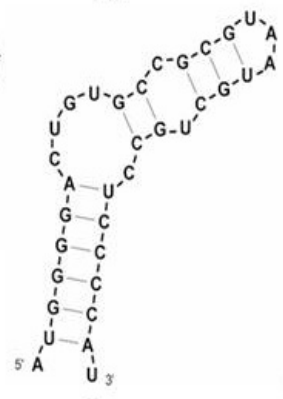

5

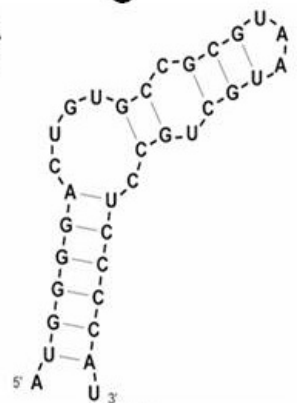

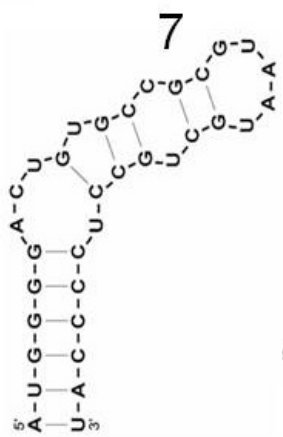
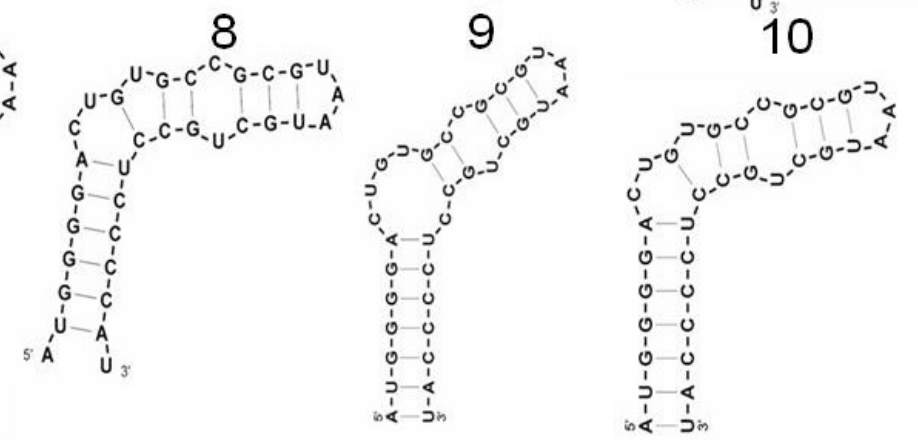

Figure 6 Possible structure predictions with double mutation. Both A10 base substitution to G10 and A12 base substitution to G12 were evaluated. Ten structures were predicted. Structures have 3 loops and strong base stem.

\section{Structure probabilities}

We also gleaned other possible information with 4 different structural pattern analyses made above. Tables 1 - 4 illustrate thermodynamic parameters for different categories of analyses that have been done in this study. The probability of all structures is also predicted, as shown in the Figures 7(a) - 7(d). In the first 3 cases (wild type, A10, and A12), the first 2 structures (1 and 2) had more probability compared to other structures (3 to 10). Moreover, structures 9 and 10 in the cases of wild and A10 base substitution had less probability. Similarly, in the case of A12, substitution structures 7 and 8 had less probability. Other structures had moderate probability. In the case of double base substitution, all the structures had similar moderate probability. 
http://wjst.wu.ac.th
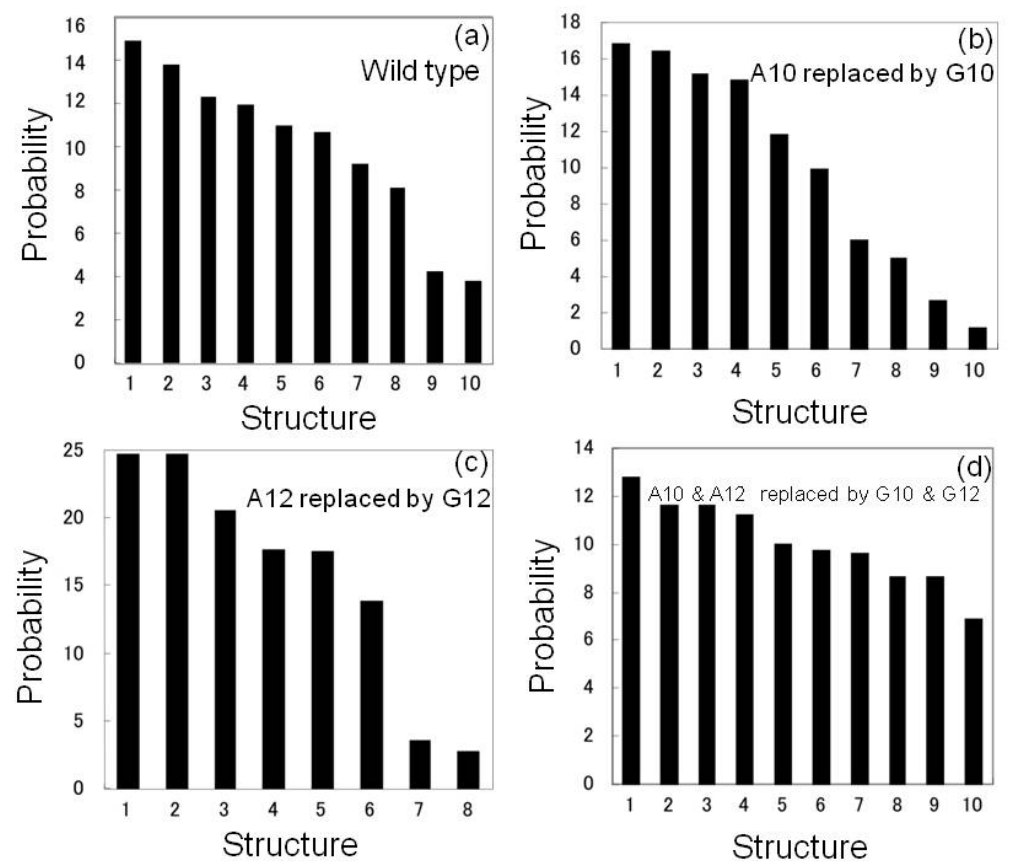

Figure 7 Representation for structural probability. (a) wild type, (b) A12 base substitution to G12, (c) A12 base substitution to G12, and (d) double (A10 to G10 and A12 to G12) base substitutions. In the cases of a-c, the first 2 structures $(1 \& 2)$ had more probability, and the last $2(9 \& 10$ or $7 \& 8)$ had less probability. In the case of (d), all have nearly the same probability.

Overall, A12 substitution has less structures and more probability compared to others. We also collected the information from dot plot (Figure 8(a)) and energy plot (Figure 8(b)) for the wild type, which represented the optimal folding, as described in the mfold web server. As reported in the present study, different structural studies $[21,27,28]$ can be implemented in a similar way with other aptamers, to be applied for sensing purposes [29-32].

(a)

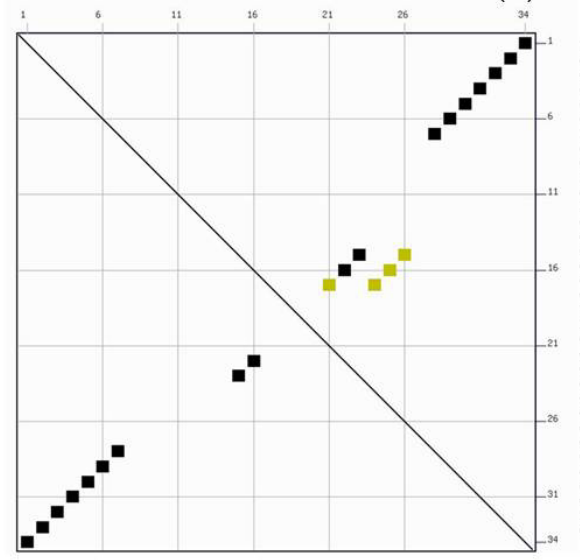

(b)

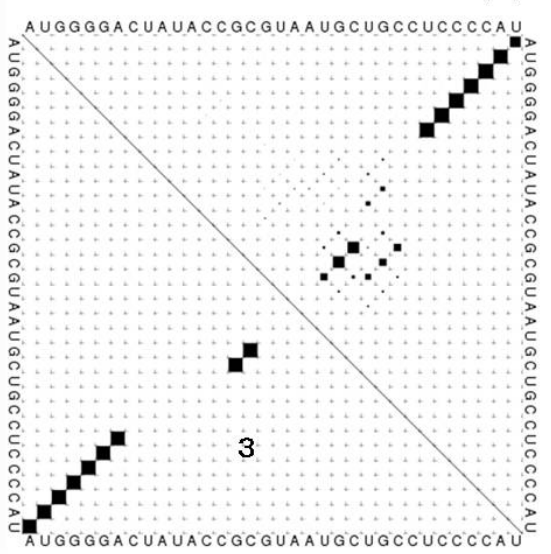

Figure 8 (a) Dot plot, (b) Energy plot form web server. Wild type is shown. Black dots represent optimal folding. Color dots represent superposition. 
http://wjst.wu.ac.th

Table 1 Thermodynamics with wild structure.

\begin{tabular}{lll}
\hline Structural element & $\boldsymbol{\delta G}$ & Information \\
\hline External loop & 0.50 & 0 ss bases and 1 closing helix \\
Stack & -1.10 & External closing pair is $\mathrm{A}^{1}-\mathrm{U}^{34}$ \\
Stack & -2.10 & External closing pair is $\mathrm{U}^{2}-\mathrm{A}^{33}$ \\
Stack & -3.30 & External closing pair is $\mathrm{G}^{3}-\mathrm{C}^{32}$ \\
Stack & -3.30 & External closing pair is $\mathrm{G}^{4}-\mathrm{C}^{31}$ \\
Stack & -3.30 & External closing pair is $\mathrm{G}^{5}-\mathrm{C}^{30}$ \\
Stack & -2.40 & External closing pair is $\mathrm{G}^{6}-\mathrm{C}^{29}$ \\
Helix & -15.50 & 7 base pairs \\
Interior loop & 4.80 & External closing pair is $\mathrm{A}^{7}-\mathrm{U}^{23}$ \\
Stack & -3.40 & External closing pair is $\mathrm{G}^{15}-\mathrm{C}^{23}$ \\
Helix & -3.40 & 2 base pairs \\
Hairpin loop & 4.50 & Closing pair is $\mathrm{C}^{16}-\mathrm{G}^{22}$ \\
\hline
\end{tabular}

Table 2 Thermodynamics of structure in which A10 is replaced by G10.

\begin{tabular}{lll}
\hline Structural element & $\boldsymbol{\delta G}$ & Information \\
\hline External loop & 0.50 & 0 ss bases and 1 closing helix \\
Stack & -1.10 & External closing pair is $\mathrm{A}^{1}-\mathrm{U}^{34}$ \\
Stack & -2.10 & External closing pair is $\mathrm{U}^{2}-\mathrm{A}^{33}$ \\
Stack & -3.30 & External closing pair is $\mathrm{G}^{3}-\mathrm{C}^{32}$ \\
Stack & -3.30 & External closing pair is $\mathrm{G}^{4}-\mathrm{C}^{31}$ \\
Stack & -3.30 & External closing pair is $\mathrm{G}^{5}-\mathrm{C}^{30}$ \\
Stack & -2.40 & External closing pair is $\mathrm{G}^{6}-\mathrm{C}^{29}$ \\
Helix & -15.50 & 7 base pairs \\
Interior loop & 3.00 & External closing pair is $\mathrm{A}^{7}-\mathrm{U}^{23}$ \\
Stack & -1.00 & External closing pair is $\mathrm{G}^{10}-\mathrm{C}^{26}$ \\
Helix & -3.50 & External closing pair is $\mathrm{U}^{11}-\mathrm{G}^{25}$ \\
Buldge loop & 3.30 & 3 base pairs \\
Stack & -3.40 & External closing pair is $\mathrm{A}^{12}-\mathrm{U}^{24}$ \\
Helix & -3.40 & External closing pair is $\mathrm{G}^{15}-\mathrm{C}^{23}$ \\
Hairpin loop & 4.50 & Closing pair is $\mathrm{C}^{16}-\mathrm{G}^{22}$ \\
\hline
\end{tabular}


http://wjst.wu.ac.th

Table 3 Thermodynamics of structure in which A12 is replaced by G12.

\begin{tabular}{lll}
\hline Structural element & $\boldsymbol{\delta G}$ & \multicolumn{1}{c}{ Information } \\
\hline External loop & 0.50 & 0 ss bases and 1 closing helix \\
Stack & -1.10 & External closing pair is $\mathrm{A}^{1}-\mathrm{U}^{34}$ \\
Stack & -2.10 & External closing pair is $\mathrm{U}^{2}-\mathrm{A}^{33}$ \\
Stack & -3.30 & External closing pair is $\mathrm{G}^{3}-\mathrm{C}^{32}$ \\
Stack & -3.30 & External closing pair is $\mathrm{G}^{4}-\mathrm{C}^{31}$ \\
Stack & -3.30 & External closing pair is $\mathrm{G}^{5}-\mathrm{C}^{30}$ \\
Stack & -2.40 & External closing pair is $\mathrm{G}^{6}-\mathrm{C}^{29}$ \\
Helix & -15.50 & 7 base pairs \\
Interior loop & 4.00 & External closing pair is $\mathrm{A}^{7}-\mathrm{U}^{28}$ \\
Stack & -3.40 & External closing pair is $\mathrm{G}^{12}-\mathrm{C}^{26}$ \\
Helix & -3.40 & External closing pair is $\mathrm{U}^{11}-\mathrm{G}^{25}$ \\
Interior loop & -0.40 & 2 base pairs \\
Stack & -3.40 & External closing pair is $\mathrm{C}^{13}-\mathrm{G}^{25}$ \\
Helix & -3.40 & 2 base pairs \\
Hairpin loop & 4.50 & Closing pair is $\mathrm{C}^{16}-\mathrm{G}^{22}$ \\
\hline
\end{tabular}

Table 4 Thermodynamics of structure in which A10 \& A12 are replaced by G10 and G12.

\begin{tabular}{lll}
\hline Structural element & \multicolumn{1}{c}{$\boldsymbol{\delta} \mathbf{G}$} & \multicolumn{1}{c}{ Information } \\
\hline External loop & 0.50 & 0 ss bases and 1 closing helix \\
Stack & -1.10 & External closing pair is $\mathrm{A}^{1}-\mathrm{U}^{34}$ \\
Stack & -2.10 & External closing pair is $\mathrm{U}^{2}-\mathrm{A}^{33}$ \\
Stack & -3.30 & External closing pair is $\mathrm{G}^{3}-\mathrm{C}^{32}$ \\
Stack & -3.30 & External closing pair is $\mathrm{G}^{4}-\mathrm{C}^{31}$ \\
Stack & -3.30 & External closing pair is $\mathrm{G}^{5}-\mathrm{C}^{30}$ \\
Stack & -2.40 & External closing pair is $\mathrm{G}^{6}-\mathrm{C}^{29}$ \\
Helix & -15.50 & 7 base pairs \\
Interior loop & 4.00 & External closing pair is $\mathrm{A}^{7}-\mathrm{U}^{28}$ \\
Stack & -3.40 & External closing pair is $\mathrm{G}^{12}-\mathrm{C}^{26}$ \\
Helix & -3.40 & 2 base pairs \\
Interior loop & 0.40 & External closing pair is $\mathrm{C}^{13}-\mathrm{G}^{25}$ \\
Stack & -3.40 & External closing pair is $\mathrm{G}^{15}-\mathrm{C}^{23}$ \\
Helix & -3.40 & 2 base pairs \\
Hairpin loop & 4.50 & Closing pair is $\mathrm{C}^{16}-\mathrm{G}^{22}$ \\
\hline
\end{tabular}


http://wjst.wu.ac.th

\section{Conclusions}

Changes in the confirmation of secondary structure are important events in the complex formation of nucleic acid and targets. In this study, a potential stable anti-FIX RNA-aptamer has been analyzed for changes in the predicted secondary structure upon complex formation using computational software. We have analyzed using the wild type and substitution of A10 and A12; our analyses predicted that involvement of A10 and A12 is important for the efficient binding of aptamer and FIX with the proper stable secondary structure. Even making a single mutation on either A10 or A12 makes changes in the confirmation with the transition of the loop region to the stem. Similar analyses can be done for other aptamers or nucleic acid to shorten the lengthier nucleic acid by gradually removing the bases from both 5' and 3' ends, using secondary structure prediction software.

\section{References}

[1] C Lozupone, S Changayil, I Majerfeld and M Yarus. Selection of the simplest RNA that binds isoleucine. RNA 2003; 9, 1315-22.

[2] SCB Gopinath, Y Shikamoto, H Mizuno and PKR Kumar. Snake-venom-derived Factor IX-binding protein specifically blocks the gamma-carboxyglutamic acid-rich-domain-mediated membrane binding of human Factors IX and X. Biochem. J. 2007; 405, 351-7.

[3] SCB Gopinath. Antiviral aptamers. Arch. Virol. 2007; 152, 2137-57.

[4] SCB Gopinath, R Wadhwa and PKR Kumar. Expression of noncoding vault RNA in human malignant cells and its importance in mitoxantrone resistance. Mol. Cancer Res. 2010; 8, 1536-46.

[5] SCB Gopinath, K Hayashi, JB Lee, A Kamori, CX Dong, T Hayashi and PKR Kumar. Analysis of compounds that interfere with herpes simplex virus-host receptor interactions using surface plasmon resonance. Anal. Chem. 2013; 85, 10455-62.

[6] T Lakshmipriya, M Fujimaki, SCB Gopinath, K Awazu, Y Horiguchi and Y Nagasaki. A highperformance waveguide-mode biosensor for detection of factor IX using PEG-based blocking agents to suppress non-specific binding and improve sensitivity. Analyst 2013; 138, 2863-70.

[7] T Lakshmipriya, Y Horiguchi and Y Nagasaki. Co-immobilized poly(ethylene glycol)-blockpolyamines promote sensitivity and restrict biofouling on gold sensor surface for detecting factor IX in human plasma. Analyst 2014; 139, 3977-85.

[8] SCB Gopinath. Methods developed for SELEX. Anal. Bioanal. Chem. 2007; 387, 171-82.

[9] SCB Gopinath, TS Misono and PKR Kumar. Prospects of ligand-induced aptamers. Crit. Rev. Anal. Chem. 2008; 38, 34-47.

[10] SCB Gopinath, K Awazu, M Fujimaki and K Shimizu. Neu5Aca2,6Gal and Neu5Aca2,3Gal receptor specificities on influenza viruses determined by a waveguide-mode sensor. Acta Biomater. 2013; 9, 5080-7.

[11] C Yang, N Spinelli, S Perrier, E Defrancq and E Peyrin. Macrocyclic host-dye reporter for sensitive sandwich-type fluorescent aptamer sensor. Anal. Chem. 2015; 87, 3139-43.

[12] X Ni, M Castanares, A Mukherjee and SE Lupold. Nucleic acid aptamers: Clinical applications and promising new horizons. Curr. Med. Chem. 2011; 18, 4206-14.

[13] SCB Gopinath. Anti-coagulant aptamers. Thromb. Res. 2008; 122, 838-47.

[14] CP Rusconi, E Scardino, J Layzer, GA Pitoc, TL Ortel, D Monroe and B Sullenger. RNA aptamers as reversible antagonists of coagulation factor IXa. Nature 2002; 419, 90-4.

[15] M Zuker and AB Jacobson. Using reliability information to annotate RNA secondary structures. RNA 1998; 4, 669-79.

[16] A Waugh, P Gendron, R Altman, JW Brown, D Case, D Gautheret, SC Harvey, N Leontis, J Westbrook, E Westhof, M Zuker and F Major. RNAML: A standard syntax for exchanging RNA information. $R N A$ 2002; 8, 707-17.

[17] M Zuker. Mfold web server for nucleic acid folding and hybridization prediction. Nucleic Acids Res. 2003; 31, 3406-15.

[18] R Knight, A Birmingham and M Yarus. BayesFold: Rational 2 degrees folds that combine 
http://wjst.wu.ac.th

thermodynamic, covariation, and chemical data for aligned RNA sequences. RNA 2004; 10, 1323 36.

[19] SCB Gopinath, D Balasundaresan, J Akitomi and H Mizuno. An RNA aptamer that discriminates bovine factor IX from human factor IX. J. Biochem. 2006; 140, 667-76.

[20] SCB Gopinath, TS Misono, K Kawasaki, T Mizuno, M Imai, T Odagiri and PKR Kumar. An RNA aptamer that distinguishes between closely related human influenza viruses and inhibits haemagglutinin-mediated membrane fusion. J. Gen. Virol. 2006; 87, 479-87.

[21] TS Kumarevel, SCB Gopinath, S Nishikawa, H Mizuno and PKR Kumar. Identification of important chemical groups of the hut mRNA for HutP interactions that regulate the hut operon in Bacillus subtilis. Nucleic Acids Res. 2004; 32, 3904-12.

[22] SCB Gopinath, K Awazu, P Fons, J Tominaga and PKR Kumar. A sensitive multilayered structure suitable for biosensing on the BioDVD platform. Anal. Chem. 2009; 81, 4963-70.

[23] SCB Gopinath and PKR Kumar. Aptamers that bind to the hemagglutinin of the recent pandemic influenza virus H1N1 and efficiently inhibit agglutination. Acta Biomater. 2013; 9, 8932-41.

[24] SCB Gopinath, Y Shikamoto, H Mizuno and PKR Kumar. A potent anti-coagulant RNA aptamer inhibits blood coagulation by specifically blocking the extrinsic clotting pathway. Thromb. Haemost. 2006; 95, 767-71.

[25] E Suenaga and PKR Kumar. An aptamer that binds efficiently to the hemagglutinins of highly pathogenic avian influenza viruses (H5N1 and H7N7) and inhibits hemagglutinin-glycan interactions. Acta Biomater. 2014; 10, 1314-23.

[26] M Zuker. Mfold web server for nucleic acid folding and hybridization prediction. Nucleic Acid Res. 2003; 31, 3406-15.

[27] SCB Gopinath, D Balasundaresan, T Kumarevel, TS Misono, H Mizuno and PKR Kumar. Insights into anti-termination regulation of the hut operon in Bacillus subtilis: Importance of the dual RNAbinding surfaces of HutP. Nucleic Acid Res. 2008; 36, 3463-73.

[28] SCB Gopinath, K Hayashi and PKR Kumar. Aptamer that binds to the gD protein of herpes simplex virus 1 and efficiently inhibits viral entry. J. Virol. 2012; 86, 6732-44.

[29] T Kumarevel, T Tanaka, M Nishio, SCB Gopinath, K Takio, A Shinkai, PKR Kumar and S Yokoyama. Crystal structure of the MarR family regulatory protein, ST1710, from Sulfolobus tokodaii strain 7. J. Struct. Biol. 2008; 161, 9-17.

[30] SCB Gopinath, K Awazu, M Fujimaki, K Sugimoto, Y Ohki, T Komatsubara, J Tominaga, KC Gupta and PKR Kumar. Influence of nanometric holes on the sensitivity of a waveguide-mode sensor: Label-free nanosensor for the analysis of RNA aptamer-ligand interactions. Anal. Chem. 2008; 80, 6602-9.

[31] V Perumal, U Hashim, SCB Gopinath, R Haarindraprasad, KL Foo, SR Balakrishnan and P Poopalan. Spotted nanoflowers: Gold-seeded zinc oxide nanohybrid for selective bio-capture. Sci. Rep. 2015; 5, 12231.

[32] SR Balakrishnan, U Hashim, SCB Gopinath, P Poopalan, HR Ramayya, M Iqbal Omar, R Haarindraprasad and $\mathrm{P}$ Veeradasan. A point-of-care immunosensor for human chorionic gonadotropin in clinical urine samples using a cuneated polysilicon nanogap lab-on-chip. PLoS One 2015; 10, e0137891. 